\title{
ARCHI-VIOLENCIA: GÉNESIS DE LA VIOLÊNCIA DE LA GÉNESIS EN LA FILOSOFIA DE DERRIDA ${ }^{1}$
}

\section{Valeria Campos Salvaterra ${ }^{2}$}

Resumen: El siguiente trabajo aborda la relación que tiene la violencia con el lenguaje y el discurso en la obra de Derrida, específicamente desde las nociones tempranas (1954-1967) de violencia originaria, archi-violencia y violencia trascendental, con el fin de abrir la perspectiva de su análisis hacia una reflexión desde el problema del origen del sentido y su despliegue textual. La cuestión de la violencia será enfocada en torno a dos aspectos de la obra de Derrida: 1) la tesis general sobre la economía en la que la violencia de origen está expuesta y se despliega, cuestión que desarrollaremos a partir de la relación teórica de Derrida con Lévi-Strauss; y 2) algunas notas más detalladas sobre la génesis del concepto de violencia, que es adoptado por Derrida en discusión directa con la tradición fenomenológica, especialmente con Husserl y Levinas. Todo, además, en el contexto de ciertas notas metodológicas sobre la herencia que no son periféricas, sino que permiten una aproximación más completa desde una idea de génesis de la violencia de la génesis.

Palabras clave: Violencia. Derrida. Génesis. Economía. Fenomenología.

Las abundantes meditaciones sobre la violencia en la filosofía occidental, incluyendo aquí a la filosofía contemporánea, han mostrado una tendencia a centrarse principalmente en el ámbito de lo político en toda su extensión. Sin intentar un análisis histórico del fenómeno de la violencia, interesa recalcar el campo de estudio al que se ha tradicionalmente subsumido como tema: la política, el derecho y la ética. La filosofía práctica, en suma. La intención de profundizar en la filosofía de Jacques Derrida se basa en la necesidad de analizar críticamente los supuestos que se asumen al hablar de la violencia desde estos marcos disciplinares, para mostrar nuevas formas de concebir un fenómeno que, si bien no puede desligarse por completo de una preocupación

\footnotetext{
${ }^{1}$ Este trabajo se realizó en el marco del proyecto FONDECYT nº11180180.

2 Profesor Asociado en la Pontificia Universidad Católica de Valparaíso (PUCV), Valparaíso - Chile. (D) https://orcid.org/0000-0002-0676-1789 E-mail: valeria.campos@pucv.cl
}

http://dx.doi.org/10.1590/0101-3173.2019.v42n4.06.p99 
propiamente ética y política, no se reduce al ámbito de la pura praxis, de la acción concreta y su inteligibilidad.

Explorar la cuestión de la violencia en la obra del filósofo argelinofrancés J. Derrida implica establecer como foco una propuesta que se acerca al fenómeno en toda su complejidad, vale decir, trascendiendo las discusiones prácticas y vislumbrando el nudo que ata a la violencia con el lenguaje, el discurso, el sentido en suma, sin por ello quedar desligada de la ley y la justicia; una violencia cuya dinámica supera y condiciona su despliegue dialéctico. Se trata de ir más allá de una concepción unívoca, reduccionista y pesimista, avanzando sobre bases que problematizan la cuestión más allá de las críticas tradicionales. Abordar el tema de la violencia desde el pensamiento de J. Derrida requiere pensar la violencia de la limitación y de la transgresión, pero también abordarla en un nivel anterior, más originario, donde se confunde con el gesto del conocimiento y del lenguaje, donde se enreda con la epopeya de la significación. Tiene que ver, ante todo, con una inquietud respecto de las condiciones de la experiencia en general, sobre cómo esta experiencia es posible y sobre las formas en que ésta tiende a desplegarse significativamente; de cómo se produce su sentido.

El siguiente trabajo se encuentra situado en el contexto de la amplia relación que la violencia tiene con el lenguaje y el discurso a lo largo de la filosofía occidental. El camino se abre específicamente desde lo que podemos llamar el rasgo ${ }^{3}$ de la violencia originaria o archi-violencia, desarrollada por el filósofo francés de modo más o menos transversal en toda su obra. El interés por asociar la violencia a la temática del origen del sentido se impuso al descubrir que el fenómeno de la violencia conserva una dimensión que no es en absoluto decidible axiológicamente; más bien, la lectura de la obra de Derrida lleva inevitablemente hacia una noción de violencia irreductible a las categorías del bien y del mal, cuya función no es sino la de una suerte de condición de posibilidad del sentido, que debe, sin duda, ponerse en cuestión en su mismo estatuto de principio. Esto se encuentra en la escritura de Derrida como una constante remisión hacia ese lugar en el origen, donde el concepto de violencia muestra la articulación misma de su carácter no-original, mucho más cercano a la noción de génesis histórica que a la de un principio arquitectónico. Lo problemático de estos caminos, de estas vías que conducen inevitablemente de ida y vuelta al y desde el "origen", impuso a este trabajo la necesidad de

\footnotetext{
${ }^{3}$ En la medida en que es una modalidad de la violencia, una suerte de determinación suya, a la vez que una rasgadura, una rotura o quiebra en el seno de aquello mismo que condiciona.
} 
delimitar su objeto de estudio a esa tan enigmática como urgente cuestión de la violencia en sus formulaciones de violencia originaria, violencia trascendental e incluso archi-violencia.

Y aunque parece fácil relacionar el problema de la violencia en Derrida con sus más tardíos escritos "ético-políticos”, ${ }^{4}$ podemos rastrear el uso del concepto desde sus tempranos trabajos sobre fenomenología. En el siguiente trabajo, nos enfocamos en la cuestión de la violencia enfatizando dos aspectos de la obra de Derrida: 1) la tesis general sobre la economía en la que la violencia de origen está expuesta y se despliega, cuestión que desarrollaremos a partir de la relación teórica de Derrida con Lévi-Strauss; y 2) algunas notas más detalladas sobre la génesis del concepto de violencia, que es adoptado por Derrida, como señalábamos, en discusión directa con la tradición fenomenológica, especialmente con Husserl y Levinas. Ambos puntos están también imbricado con algunas consideraciones metodológicas que considero son insoslayables a la hora de abordar la filosofía de Derrida, pues son además estructurales para lo que se ha llamado "la deconstrucción". Ellas dicen relación con la idea de una "génesis" de el concepto de violencia originaria o genética, tomando de aquí su título nuestro trabajo -génesis de la violencia de la génesis. Por esta razón, la exposición comenzará invirtiendo la progresión aquí seńalada, comenzando por estas últimas consideraciones para luego retornar al orden propuesto.

\section{GÉNESIS DE LA VIOLENCIA DE LA GÉNESIS: LA LÓGICA HEREDITARIA}

¿Es Derrida un crítico de la violencia? Esta es una pregunta difícil de abordar, pues la respuesta podría ser tanto afirmativa como negativa, según se aborde la filosofía del autor. ${ }^{5}$ En cualquier caso, crítico o no, parece claro

\footnotetext{
${ }^{4}$ Sostenido entre otros por Robert Bernasconi (1991), Drucilla Cornell (1992) y Simon Critchley (1999a y b). Derrida dice en Canallas (2005, p. 58) que "jamás hubo, en los años ochenta o noventa, como a veces se pretende, un political turn o un ethical turn de la 'deconstrucción' tal y como, al menos, yo la he experimentado [...] Sencillamente, lo que haya pasado no tiene ninguna relación, ninguna semejanza con lo que la figura del turn, de la Kehre, del giro o de las tornas, podría hacer que nos imaginásemos."

${ }^{5}$ Quizás, lo primero que se deba responder es no: no, en la medida en que no hay en sus textos una intención crítica estricta, un gesto manifiesto de krinein guiado por una cierta ley del género. Por el contrario, lo que se encuentra frecuentemente es una puesta en cuestión del krinein mismo: si la violencia es "trascendental" -cuestión que seguiremos y mostraremos en las siguientes páginas-, no puede haber un área no violenta del discurso. Dado que 'crítica' viene de la palabra griega krinein -cortar, separar, dividir, trazar un límite- no puede haber lugar para una crítica de la violencia que
} 
que Derrida es un pensador de la violencia. Y, sin embargo, tampoco parece descabellado afirmar -desde otra perspectiva- que Derrida no tiene en sentido estricto una "filosofía" de la violencia. No hace de la violencia un tema directo de sus meditaciones ni tiene una tesis explícita sobre ella. Esto no es excepcional, pues sucede con todos los conceptos centrales del pensamiento de Derrida, tal como confiesa a uno de sus entrevistadores:

Usted tiene razón, no hay una filosofía del tiempo sobre la que yo haya escrito. Pero no hay una filosofía del acontecimiento tampoco, o de la muerte. No hay filosofía de nada. Yo he, en efecto, comenzado por trabajar la herencia filosófica en cuanto al tiempo Kant, Husserl, Heidegger sobre todo-y el privilegio de la presencia en el pensamiento del tiempo. (DERRIDA, 2004). ${ }^{6}$

La violencia aparece, así, como muchos de sus temas, en un análisis de la tradición filosófica, a partir de una aproximación metodológica que proponemos llamar "lógica de la herencia": una lectura dinámica en la cual, a través de un diálogo crítico con distintos pensadores de la tradición -de Platón a Marx, de Lévi-Strauss a Benjamin, entre muchos otros- Derrida, a la vez, pone en cuestión y refuerza algunas de las ideas que están en juego en sus análisis. En términos que el mismo Derrida utiliza con frecuencia, su trabajo deconstructivo de la tradición es en realidad una forma de ser fiel a ella y no simplemente una aproximación crítica-destructiva, poco generosa. ${ }^{7}$ En efecto, para Derrida, ser fiel y generoso en el análisis de un autor implica explorar su texto muy cuidadosamente, sin juzgarlo a partir de un ejercicio basado, en última instancia, en estructuras normativas externas. Por lo anterior, la propuesta de análisis del problema de la violencia implica rastrear su configuración en "escenas de herencia" o incluso "escenas de génesis": configuraciones textuales en que Derrida construye su propia filosofía siempre

la separe de la no-violencia. Más que pensar en términos de una dicotomía violencia/no-violencia, Derrida sostiene que debemos pensar la violencia como una economía (DERRIDA, 1967a, p. 172).

${ }^{6}$ Cf. Nielsberg (2004): http://www.humanite.fr/node/299140

7 La relación entre Derrida y su herencia puede describirse como una amistad infiel o como una fidelidad en el corazón de la enemistad, porque la herencia es siempre fiel-infiel "infiel para ser fiel ("infiel para ser fiel: en vistas de ser fiel y, al mismo tiempo, porque es o será fiel)" (DERRIDA, 2008b, p. 219). Porque el amigo que soy yo para mí mismo es también el enemigo. Porque en el "entre" dos es que se juega la imposible separación de la amistad y la enemistad pues, siguiendo a Ch. Ramond, la preposición "entre" separa y relaciona a la vez: "no hay nada entre nosotros" puede significar tanto "no hay relación entre nosotros, no hay proximidad" como "somos tan cercanos que nada no separa" (RAMOND, 2007, p. 55). 
en relación con el pensamiento del otro, como recibiendo una suerte de herencia. Esta aproximación supone que hacer filosofía depende de un proceso de formación dado en relación con otro, con muchos y múltiples otros insertos también en múltiples descendencias en las que se traspasa más de un legado. Ser es, entonces y en sentido fuerte, ser generado y, con ello, ser parte de una generación: ser es heredar. "El ser de lo que somos es, primariamente, herencia, aunque lo queramos y lo sepamos o no" (DERRIDA, 1993a, p. 94), dice Derrida en un texto tardío, Espectros de Marx.

No hay pues, un origen, o una originalidad absoluta desde el que esta filosofía podría pensarse, sino todo lo contrario. Por ello, hay que poner también bajo una suerte de tachadura la noción de "lógica" que utilizamos junto a la palabra herencia. Pues la herencia no está inserta en una sola y unívoca "línea" de descendencia que produciría un solo legado: el genus, el género, la familia, formada mediante un traspaso, un testimonio que se envía de una generación a otra, de un miembro del linaje a otro, de un padre a un hijo y de este también a los suyos. Este sería el linaje que constituye la filiación, el dominio del padre y la herencia de los hermanos, y es justamente una noción de herencia que debe ponerse en cuestión. Pues decir que Derrida está atado a ciertas "generaciones" de filósofos puede inducir a confusiones, principalmente porque Derrida no fue nunca un filósofo dado a establecer relaciones de exacerbada admiración ni con sus antecesores, ni con la tradición, pero tampoco dado a establecer relaciones de intimidad con sus pares (PEETERS, 2013); por ello, no podríamos hablar de relaciones de "familia" establecidas entre sus "amigos"; no podríamos decir nunca que Derrida tuvo "hermanoscolegas" y, menos aún, habremos de referir a un "padre" o una "madre" de su filosofía -menos entonces, podemos llamarlo el "padre" de la deconstrucción. ${ }^{9}$ Si decimos que es necesario insertar la filosofía de Derrida, y específicamente aquí la cuestión de la archi-violencia, en una línea generacional, lo decimos de otro modo que como puede primaria y fundamentalmente interpretarse. Porque la herencia para Derrida excluye, paradójicamente, todo vínculo de familia. No se hereda por la sangre, por pertenecer a una familia, por tener hermanos que comparten el haber nacido de un único padre, que sería el principio de unificación de toda casa, el tronco de todo árbol genealógico. Por el contrario, para Derrida heredar es justamente lo que no se da a partir del

${ }^{8}$ Cf. Derrida (1967b, p. 38). Derrida recurre a la tachadura por referencia a Heidegger. La llama, en el texto seńalado, "la última escritura de una época", pues "bajo su trazo se borra, quedando legible, la presencia de un significado trascendental".

${ }^{9}$ Como lo hace, por ejemplo, J. M. Garrido en Chances de la pensée (2011, p. 108). 
lazo consanguíneo, lo que excluye cualquier identidad de código genético. Y por eso también una herencia y un legado no suponen nunca la homogeneidad de lo trasmitido y de lo generado, sino, por el contrario, su heterogeneidad:

Consideremos, primero, la heterogeneidad radical y necesaria de una herencia $[\ldots]$ se hereda siempre de un secreto -que dice: "léeme ¿serás alguna vez capaz de hacerlo?”. La elección crítica requerida por toda reafirmación de la herencia es también, como la memoria misma, la condición de la finitud. El infinito no hereda, no se hereda. (DERRIDA, 1993a, p. 40).

Si la herencia es heterogénea, si en el origen de la generación hay siempre más de uno, relación con lo que no es Uno, con lo que no es lo mismo, entonces nunca realmente se sabe lo que se hereda ni de quién, es decir, no hay conocimiento certero de la herencia. De ahí la necesidad de una puesta en cuestión insoslayable de la herencia, pues no se puede sin más aceptarla o traspasarla, pues nunca se sabe realmente qué es lo que se acepta o traspasa. Espectros de Marx refuerza esta idea a partir de la introducción de un principio de desestabilización de la herencia en cuanto lineal y continua, justamente a partir de la entrada del espectro que viene a penar y a hacer temblar la estructura general de la herencia y las generaciones. ${ }^{10}$ Las generaciones son en realidad descendencias espectrales, de las que no se puede determinar su status vital -si están vivas o muertas- y es por eso que no pueden clausurarse ni homogeneizarse más que a contrapelo. Si esta situación puede constituir una suerte de "lógica" tanto de la filosofía misma de Derrida como de su

\footnotetext{
${ }^{10}$ Siempre es posible referir el problema del espectro al problema general del tiempo. La espectralidad, como lo que no se deja reducir al esquema presencia/ausencia, fenomenalidad/nno-fenomenalidad, supone pensar un tiempo que no es sino un diferir originario, un desajuste primero que imposibilita una experiencia unitaria, unívoca y autoidéntica. En el texto de 1993, Espectros de Marx, Derrida acuña la expresión shakesperiana "the time is out of joint", frase que hace alusión a un tiempo desarticulado, dividido, desencajado, dislocado, trastornado, acosado, perturbado, a la vez desarreglado y loco (DERRIDA, 1993a, p. 42). A un tiempo fuera de sus goznes, fuera de su eje de sincronía y que, por tanto, no se puede controlar, no se deja dominar ni ordenar, pues excede y sobrepasa violentamente todo intento de sincronización. Es un tiempo anacrónico respecto de sí mismo, que se resiste a quedar reducido en la linealidad de un armónico transcurso siempre progresivo, como tampoco se deja fijar, aislar o analizar desde la puntualidad estática en que se fundamenta un "presente vivo". Esa violencia, esa fuerza del exceso del tiempo respecto de sus categorías metafísicas [el ahora -nun-, el punto, la línea, el círculo, el presente, etc., cf. Derrida (1972, p. 31-73)] no tiene lugar, es constantemente reducida por el pensamiento, justamente en la medida en que torna imposible toda fijación de esencias y, por tanto, toda determinación absoluta. De ahí que su violencia sea siempre amenazante, acaso injusta, y es el mismo Hamlet quien ya contrapone el out of joint al "estar derecho del tiempo", "en el buen derecho o en el camino derecho de lo que marcha bien” (DERRIDA, 1993a, p. 45).
} 
lectura, es precisamente porque estas generaciones espectrales no aparecen sino en el lenguaje, y en el lenguaje en cuanto escritura: "No puede hablarse de generaciones de calaveras o espíritus (Kant qui genuit Hegel qui genuit Marx) más que por la condición de la lengua -y la voz, en todo caso de lo que marca el nombre o está en su lugar." (DERRIDA, 1993a, p. 30). El lugar de la lengua y del nombre es el lugar de la escritura y, por ello, el lugar del otro. Porque no hay propiedad natural de la lengua ${ }^{11}$, siempre se genera por expropiación, por una suerte de alienación "originaria”, por una salida fuera de sí en sí, anterior a la constitución de un "adentro" o de una "mismidad" cerrada. Todas estas ideas, que se concentran en la noción de escritura o archi-escritura ${ }^{12}$, son las que hay que pensar para aproximarse a la filosofía derridiana, y son todas cuestiones de herencia, de relación, de filiación sin filiación, de amistad como enemistad. Pues si la lengua es siempre la lengua del otro radical, del otro irreductible a mí mismo, entonces la herencia no es nunca familiar, no está gobernada por una normatividad patronímica, no está clausurada por una igualdad de código ni está determinada por una pura fidelidad irrenunciable.

Se ve ya como en el tema de la herencia se mezclan todas las problemáticas derridianas, la de la relación con otro, el secreto, la muerte, la ley, la crítica o la violencia. Por ello, si el origen es una génesis, entonces no hay sino violencia de la génesis, violencia como génesis: choque, guerra, tensión, conflicto. La tesis entonces de una violencia originaria como violencia genética no puede ser abordada sino a través de una génesis de esa violencia, que implica acudir a la cuestión de la herencia y del heredero, es decir, ser-uno-ya-como-otro, no ser uno más que como otro, en relación con otro: "En el fondo, de una apelación a otra, es el mismo el que habla, él, yo, y el lenguaje libera esta sustitución: 'yo' es yo, pero un 'yo' es un 'él'. El uno es el otro. El uno guarda y se guarda del otro. El uno se hace violencia.” (DERRIDA, 1994, p. 78).

Es justamente esta violencia como violencia de la génesis la que está en el centro de este trabajo, y la abordaremos a partir también de un proceso de génesis: génesis de la violencia de la génesis, podría ser otro nombre

\footnotetext{
${ }^{11}$ Cf. El monolingüismo del otro (1997c).

${ }^{12}$ La tesis de la archi-escritura supone que los atributos del signo gráfico dependen no solo del acto intencional que lo trae a la vida; sino también, y con mayor fuerza, del material y la naturaleza finita de la inscripción gráfica. En consecuencia, el signo opera en cada texto como un testimonio y un testamento de la muerte del sentido trascendental y de la subjetividad (DERRIDA, 1993b, p. 107). La distancia insalvable entre el signo gráfico y el espíritu (mente, alma, intelecto, intenciones, etc.) que formaliza su elemento conceptual es el componente clave para entender la escritura como un tema problemático para la metafísica tradicional o para las teorías trascendentales de significado.
} 
de este artículo. En lo que sigue, abordaremos la relación de herencia que Derrida guarda con Husserl y Levinas en torno al tema de la violencia, mostrando así cómo es la relación de lectura pausada de sus textos la que configura el concepto de violencia originaria, relación que, como decíamos, si bien es deconstructiva, no es del todo destructiva: pues de genus también viene generositas lo que hace el generosus, el que es noble por el origen. La generosidad es lo propio de las generaciones, de los linajes, lo que se hace con y por la herencia. Pero, habíamos dicho, si la herencia es múltiple, si siempre es más de una, y si el linaje, si las generaciones no pueden reconducirse a un solo y único origen, a un padre pleno y presente, entonces la generosidad no implica unidireccionalidad del don. Dar de modo lineal, recto, con origen y destino definido, dar a un hermano, hijo de un mismo padre, un igual a mí, un idéntico, es justamente lo que la generosidad no es. El dar de la generosidad, si es que esa es la actitud de las generaciones, es un dar equívoco, que se envía sin destinatario asignado, sin saber dónde llegará - si llega ${ }^{13}$. Se da en generosidad pero no se sabe a quién, pues no hay unidad ni vínculo conocido entre el que da y el que recibe. Si es así, debemos seguir a Gerard Granel -en un texto tan temprano como lúcido-, cuando dice que la generosidad es la guerra, la tensión con el otro que nunca se resuelve en la unidad de la consanguineidad: "Es que entonces la misma generosidad, si no es enteramente el medio de la guerra ( $y$ tal vez en efecto es -o mejor, después de todo- a veces la declaración misma de la guerra), corre el riesgo de hacer que todo caiga en una horrible calma, de suerte que a final de cuentas no saldríamos nunca de una biblioteca universitaria." (1972, p. 174). Generosidad y violencia no están, entonces, nunca en contradicción la una con la otra.

\section{ECONOMÍA DE LA VIOLENCIA}

La violencia, como se ha sugerido, es tratada por Derrida en el contexto de la noción de economía, pues la fórmula "economía de la violencia" es

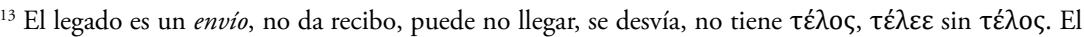
secreto indescifrable del crimen de la herencia, ese lugar-tiempo donde hay que habérselas siempre con el otro, en su exterioridad y singularidad radicales, es lo que fuerza la decisión, es la inyunción misma que manda aquí y ahora. Esto tiene que ver con la singularidad o unicidad de ese instante del legado. Pues aunque el mandato mande dar respuesta al otro y, con ello, es la forma trasferencial del don lo que se pone en juego -la indeterminabilidad de su envío y la heterogeneidad del "remitente"- "un don tal tendría que ir derecho a lo único, a lo que su nombre habrá nombrado únicamente, a eso único que habrá dado su nombre. Ese derecho no depende de ningún derecho, de ninguna jurisdicción trascendente al don mismo." (DERRIDA, 1987, p. 155).
} 
probablemente la más frecuentemente utilizada para referir al problema de la violencia en los textos de Derrida. Siguiendo la lógica de la herencia situada en el punto anterior, la idea es originalmente formulada en el texto dedicado a Levinas, Violencia y metafísica, de 1967, para ser completamente problematizada en una escena de diálogo con Lévi-Strauss en De la gramatología, publicado el mismo año. En ambos escenarios, la idea de una economía de la violencia apunta a una estructura dinámica de fuerzas y flujos que están en constante circulación, inter-relación, cambio, sustitución, referencia, capitalización, retorno, etc. (DERRIDA, 1991, p. 17-18). ${ }^{14}$ La violencia es así entendida como un fenómeno dinámico de compleja estructura, compuesta de movimientos plurales y multidireccionales representados por la figura de la economía, que incluye también múltiples quiebres internos. Es por esta complejidad que es imposible crear una "tipología" de la violencia que no esté determinada y, por esta razón, simultáneamente subvertida por los caóticos desplazamientos de la economía.

Y sin embargo, a partir de los análisis y lecturas deconstructivas del texto de Lévi-Strauss, Derrida propone una "triple estrutura" de la violencia (1967b, p. 164-165). Esta es la primera aproximación a una posible sistematización del concepto, en la medida en que Derrida establece allí una bien definida tipología de al menos tres tipos diferentes de violencia. Siempre en el contexto del análisis del texto de Lévi-Strauss, Tristes trópicos, Derrida dice lo siguiente:

Habría, en efecto, una primera violencia en el nombrar. Nombrar, dar los nombres que estaría eventualmente prohibido pronunciar, tal es la violencia originaria del lenguaje que consiste en inscribir en una diferencia, en clasificar, en suspender el vocativo absoluto. Pensar lo único en el sistema, inscribirlo en él, tal es el gesto de la archi-escritura: archiviolencia, perdida de lo propio, de la proximidad absoluta, de la presencia a sí, perdida en verdad de lo que jamás tuvo lugar, de una presencia a sí que no ha sido jamás dada sino sońada y siempre ya redoblada, repetida, incapaz de aparecer de otra modo que en su propia desaparición. A partir de esta archi-violencia, prohibida y por tanto confirmada por una segunda violencia reparadora, protectora, que instituye la "moral", que prescribe

\footnotetext{
${ }^{14}$ En este texto, Derrida se pregunta: ¿Qué es la economía? (Qu’est-ce que l'économie?). Y su respuesta es (cito original francés): "Parmi ses prédicats ou ses valeurs sémantiques irréductibles, l'économie comporte sans doute les valeurs de loi (nomos) et de maison (oikos, c'est la maison, la propriété, la famille, le foyer, le feu du dedans). Nomos ne signifie pas seulement la loi en général, mais aussi la loi de distribution (nemein), la loi du partage, la loi comme partage (moira), la part donnée ou assignée, la participation. Une autre sorte de tautologie implique déjà l'économique dans le nomique comme tel. Dès qu'il y a loi, il y a partage: dès qu'il y a nomie, il y a économie. Outre les valeurs de loi et de maison, de distribution et de partage, l'économie implique l'idée d'échange, de circulation, de retour."
} 
la ocultación de la escritura, la borradura y la obliteración del nombre que se dice propio que ya dividía lo propio, una tercera violencia puede eventualmente surgir o no surgir (posibilidad empírica) en lo que llamamos corrientemente el mal, la guerra, la indiscreción, la violación: que consiste en revelar por efracción el nombre que se dice propio, es decir, la violencia originaria que ha despojado a lo propio de su propiedad y de su pureza (propreté). Tercera violencia de reflexión, podríamos decir, que desnuda la no-identidad nativa, la clasificación como desnaturalización de lo propio, y la identidad como momento abstraído del concepto. En este nivel terciario, el de la conciencia empírica, es que debería sin duda situarse el concepto común de violencia. (DERRIDA, 1967b, p. 164-165).

Una primera violencia es llamada "originaria", y es adjudicada al lenguaje, especialmente a la operación del nombrar como clasificación de lo singular o "inscripción en una diferencia". ${ }^{15}$ Derrida también escoge llamarla aquí "archi-violencia", en la medida en que es la violencia tomada como una suerte de condición de posibilidad del nombrar que, en cuanto clasificatorio, opera como estructura de la significación en general. Un segundo nivel puede ser llamado "violencia de la ley" o "violencia moral" y es referido al gesto de la prohibición. Específicamente, en cuanto prohibición de la primera violencia o violencia originaria. Es lo que sucede en la sociedad Nambikwara descrita por Lévi-Strauss, donde está prohibido proferir el nombre propio. Una tercera violencia es llamada "violencia en sentido corriente", o "violencia empírica", y refiere a una suerte de contra-violencia sobre el segundo nivel de violencia; una cierta violencia de transgresión de la ley que siempre puede ser juzgada de "mala" o "peligrosa". Por supuesto, este tercer nivel de violencia, dice Derrida, es el más visible de todos $-\mathrm{y}$ es por eso que lo llama "violencia en sentido corriente”. Es, en efecto, el único que puede ver Lévi-Strauss, y es por eso que a la vez que declara la bondad originaria de los Nambikwara seńala al extranjero $-\mathrm{y}$ a lo que éste trae desde fuera: la escritura- como origen del mal. ${ }^{16} \mathrm{El}$ segundo nivel, la violencia de la ley, no es vista por Lévi-Strauss aún

\footnotetext{
${ }^{15}$ Esta descripción está tomada de lo que el mismo Lévi-Strauss determina como el gesto propio del nombrar: asignar al otro, al singular, a una clase. Derrida cita al antropólogo: "No se nombra jamás: se clasifica al otro." (1967b, p. 160, nota 6).

${ }^{16}$ La deconstrucción que opera sobre el texto de Lévi-Strauss tiene como fin, en términos generales, mostrar la denigración que lleva a cabo el antropólogo respecto de la escritura, asociándola con una violencia que sería no sólo la única, sino además unívoca. Esta violencia estaría definida por una constatación histórica de la que la escritura es la condición general de la explotación del hombre por el hombre (p. 173 y ss.), y estaría marcada, signada y desencadenada, por la llegada del extranjero (166167). Estas concepciones serían, para Derrida que tomará muchas páginas en mostrarlo, señales de una nostalgia roussoniana operando en el trabajo de Lévi-Strauss, y en última instancia darían cuenta de
} 
como una forma clara de violencia, pues es la condición de ese orden social que describe, justamente, en términos de no-violencia. Sin embargo, y aunque sea en general menos visible, ha sido ampliamente abordado críticamente por una cierta tradición -en la cual W. Benjamin es uno de los autores clave ${ }^{17}$ como un gesto de corte agresivo y violento entre lo bueno y lo malo, lo legal y lo ilegal, sin ningún otro fundamento que la fuerza ínsita en el corte mismo. Derrida lo describe en otro lugar como la respuesta necesaria ante la falta de origen o fundamento para el sentido de la ley en general, tomando por ello la forma de una tautología performativa (DERRIDA, 2008a, p. 86). Y aun cuando Derrida es tanto un lector respetuoso y un heredero de Benjamin, cualquier filosofía que, ya se inscriba en esta tradición violenta, ya la critique sin ver la completa y compleja estructura económica de la violencia, cae en lo que proponemos llamar visión jurídica de la violencia (CAMPOS, 2013, 2017). ${ }^{18}$ Esta visión, que es sostenida por casi todas las críticas de la violencia no abarca el fenómeno en toda su extensión, esto es, no reconoce la dinámica de la violencia desde el punto de vista de la economía.

Y aunque Derrida habla de modo explícito de una "estructura" de la violencia, una lectura no demasiado cuidadosa puede generar, sin duda, una irresistible tentación: la de ver ahí, en este lugar particular y único, un esbozo o una síntesis de toda la economía de la différance en cuanto atravesada por la violencia. Así lo hace Richard Beardsworth (2008), quien -entre otros [como también (MARSH, 2009)] - postula que es en este párrafo donde se juega toda la tesis sobre la violencia en Derrida. Y es que no es difícil ver aquí una división que se puede también encontrar en las tesis mismas de Derrida sobre la filosofía en general: una violencia de la archi-escritura o de la archi-huella que sería "prohibida", escondida, negada, por una violencia de la ley que, en último término, no podría escapar del exceso mismo de esa violencia que fue suprimida en primer lugar, de una violencia de transgresión de la ley que puede adoptar diversas formas y que, por ello, no es sino contingente en su despliegue. Esta estructura sería, entonces, la de la economía en general de la

la homogeneidad con que la violencia es descrita por la tradición filosófica, en estrecha relación con la escritura.

${ }^{17}$ Cf. BENJAMIN, W. "Para una crítica de la violencia". Traducción y notas de Pablo Oyarzún. En Oyarzún P., Pérez C. Y Rodríguez, F. Letal e incruenta. Walter Benjamin y la crítica de la violencia. Santiago: LOM, 2017.

${ }^{18}$ En dichos textos, muestro que es mediante una cierta noción de lo jurídico que la violencia puede volverse primordial para la deconstrucción derridiana. Dicho concepto debe rastrearse en el lazo de Derrida con Husserl y su descripción de lo jurídico en la fenomenología. 
escritura en los términos en que la piensa Derrida y abarcaría, por tanto, la totalidad de su obra, en sus distintos lugares y épocas. Sin embargo, nuestra tesis rechaza esta simplificación de la cuestión. Si bien, esta estructura triple de la violencia representa de manera bastante precisa los posibles niveles de lo que sería la "economía" de la différance en Derrida, creemos que hay que guardar cautela. Pues si bien nos puede servir para mostrar la compleja trama que hace de la violencia una estructura económica en el sentido antes mencionado, esta estructura opera también como una trampa analítica que aborda la cuestión de la crítica de la violencia en términos de su visibilidad: de lo que se trata es de mostrar aquello que ciertas críticas, por más lúcidas que sean, no pueden realmente llegar a ver. Sin querer profundizar en esto último, lo que recalcamos es que, para abordar de modo más amplio la cuestión de la economía asociada a la violencia se debe evitar caer bajo el encanto seductor de esta tríada y tener en cuenta:

1. La dificultad que implica fijar esos momentos en una estructura, justamente porque si bien se puede decir a priori que la archi-escritura como violencia es originaria y constituyente, no se pueden determinar con certeza las consecuencias ni los efectos empíricos concretos de ese a priori, y menos aún el modo en que dichos efectos se articulan para tener lugar. En otras palabras, su a priori no puede ser estrictamente condicionante - no puede clausurar las posibilidades de sus derivas-, por lo que su trascendentalidad no puede ser total. En efecto, la archi-escritura como violencia trascendental -como la llama Derrida en Violencia y metafísica- es justamente lo que permite "abrir el juego" del sentido - del pensamiento y del lenguaje-, pero en la medida en que dicha condición de apertura es ya siempre violenta, las posibilidades que condiciona no se dejan nunca determinar hasta el límite: he ahí la contaminación entre lo trascendental y lo empírico que Derrida ya constata en la fenomenología husserliana, a partir de la cual se organizará el "método" de su filosofía. En cuanto principio trascendental-empírico, la différance puede ser descrita -y quizás no puede sino ser descrita- en términos de fuerza o de violencia: justamente porque la fuerza es lo que por definición no se deja determinar, no se deja fijar en una forma (eidos, morphé), es decir, en la presencia plena de un principio trascendental puro -ya sea histórico o estático. 
2. La constatación de que, en cuanto economía, la estructura de la violencia es ella misma un diferencial, en la medida en que se articula difiriendo de sí misma en estos tres niveles. En efecto, Derrida -como lo nota agudamente Catherine Malabou (1990)- parece señalar, en un momento de esta misma estructura, una cierta diferencia jerárquica que habría entre la archi-violencia y la "violencia en sentido corriente", que cuestionaría la lógica misma de la huella como lo que oblitera todos los sentidos propios, a la vez que lo propio del sentido. Malabou sugiere la posibilidad de que tal división implique a su vez la posibilidad de la reducción de lo que Derrida llama "violencia empírica" a la "violencia trascendental" del primer nivel, es decir, la delimitación estricta de un campo de "verdadera violencia" (MALABOU, 1990, p. 320; DERRIDA, 1967b, p. 166) -trascendentalque dejaría fuera toda violencia derivada -empírica. Sin embargo, como lo hemos esbozado, se sigue de las múltiples aclaraciones que hace Derrida sobre las nociones de "archi" y de "trascendental" -como siempre sometidas a tachadura- que esto no debe entenderse así. Por lo que sigue en el texto de la gramatología, se entiende que ese "sentido corriente" es en realidad un cierto pliegue interno de la economía de la violencia y la contra-violencia, esto es, una violencia esencialmente ligada al movimiento obliterante de la archi-violencia y nunca simplemente derivada. Nunca se afirmará de modo directo su calidad de "sentido corriente" versus un "sentido propio", lo que implica que esta no se podría ni derivar, ni reducir. En efecto, es a Lévi-Strauss a quien se apunta como causa de esta división: él es quien que ve a esta violencia del golpe, violencia física o empírica entre las niñitas -"guerra en sentido corriente"- no solo como la única violencia, sino también como un accidente sobrevenido desde el exterior a un terreno de inocencia y bondad natural, producido ciertamente por la presencia del extraño, del observador (DERRIDA, 1967b, p. 166), en lugar de algo inherente al fenómeno general de la violencia como archi-violencia misma del nombrar. Derrida pone en cuestión la actitud del etnólogo que sitúa la escena de los nombres propios solo a nivel de violencia empírica, como una violación y una catástrofe que afectarían solo accidentalmente a un estado de paz anterior, no solo como si fuese el único nivel, la única forma de violencia, sino que además como siempre totalmente injusta. Derrida muestra que esa violencia empírica es en realidad interior a la archi-violencia como violencia en general, porque esta violencia empírica no nombra sino una cierta violencia de transgresión, de transgresión de la ley metafísica, de la vigilancia policial. Es una violencia de contra-golpe o, 
más bien, es la misma violencia originaria desbordando -en su movimiento de suplantación- los límites del texto metafísico, del texto de la ley y de la ley del texto, sea esta ley puramente simbólica, sea escrita, empírica, totalmente situada y actual. Toda transgresión a posteriori escenifica la trasgresión a priori de la ley del texto, la ley que solo puede ser obedecida mediante la transgresión anticipada. Además, hay que notar que Derrida llama a esta violencia "empírica" justamente porque puede surgir o no surgir, porque su posibilidad es empírica. Pero esa posibilidad empírica -lo hemos visto ya y seguiremos en ello- es estructural, es la estructura misma de la violencia diferancial: archi-violencia invaginada, auto-repliegue de la violencia sobre sí misma que la hace sobreponerse a sí en sí. Este repliegue de lo exterior en lo interior, de lo supuestamente exterior a la violencia al interior de ella, la hace ser creadora $y$ destructora, juste $e$ injusta, fuerte $y$ débil, etc. Es solo en ese double bind de la violencia-signado por el $y$ de un al mismo tiempo y nunca por una mera contradicción dialéctica- que está en jugo la posibilidad empírica de la violencia como estructuralmente contenida en la violencia trascendental o archi-violencia y, por tanto, no puede ser totalmente aislada de esa complicación diferencial. De ahí que tratar esta estructura separando sus niveles, intentando hacer coincidir cada nivel con los movimientos que hemos señalado, supondría establecer ciertos cortes y límites cuya legitimidad podría ser cada vez entredicha, es decir, cada vez deconstruída por una apelación a la misma violencia y a la ley del texto o del relato que dichos límites intentarían contener y reducir.

Estos reparos o cauciones señalan que hay que pensar la economía, que la noción de economía es lo fundamental si hay que abordar la estructura completa, a partir de su posibilidad. La economía de la violencia apuntaría justamente a ese desajuste de lo propio en sí mismo, a su diferencia y diferir constitutivo y constituyente respecto de sí. Eco-nomía: ley de la partición, intercambio, circulación; pero más fundamentalmente, ley del hogar, del oikos, de lo familiar. Ley de lo en-lo-de-sí, ley de lo propio. Si hay economía -oikos-nomía- de la violencia, hay una ley de lo propio que es una ley de la violencia. ¿En qué consiste esta ley de lo propio como norma de la violencia? Aquí es donde es necesario, volver a escritos anteriores de Derrida. Para ello, es necesario en efecto enfocarse en el primer estadio de la "estructura", la operación diferencial del lenguaje que Derrida también identifica con la escritura como archi-escritura. Esta violencia es la que tiene un gran rendimiento en la 
filosofía derridiana pues es el punto clave para entender la diferencia entre una visión económica de la violencia y una jurídica. Las tesis sobre al archiescritura, como decíamos, son cruciales para la idea de una violencia originaria o una archi-violencia, pues es una violencia que pertenece al lenguaje mismo y a su operación conceptualizadora. Aquí es donde se encuentra, entonces, el insoslayable vínculo con la fenomenología.

\section{Fenomenología y Violencia: entre HuSSERL y LeVinaS}

El concepto de violencia originaria se "impone" -por cálculo histórico y efectividad teórica, quizás- en el primer período de escritura de Derrida (19571972), a partir de discusiones con la fenomenología de Husserl en relación con el problema de la génesis en la fenomenología, lugar desde donde aparecen para Derrida todos sus problemas. Problemas pero, también y extrañamente, todas sus posibilidades. El tiempo abordado desde la génesis del sentido ${ }^{19}$ es, sin duda, el poder-límite de la textualidad fenomenológica, aquello desde lo que la fenomenología es posible, pero también lo que la hace imposible. El descubrimiento de esta dimensión auto-contradictoria de la fenomenología guía a Derrida hacia lo que será su "único" tema, o el tema ubicuo a todas las reflexiones hereditarias: la cuestión del doble vínculo, de la contaminación de los opuestos, de la imposibilidad de la lógica de la plena contraposición (1990: VI-VII). El tiempo en la fenomenología, con todo lo que tiene de complejo su análisis, es lo que abre para Derrida estas cuestiones. Esto se puede rastrear en las tempranas críticas a Husserl (El problema de la génesis en la filosofía de Husserl, de 1956) sobre la imposibilidad de reducir el tiempo mundano al tiempo sincrónico de la conciencia a partir del presente como modo fundamental de la síntesis temporal. De modo esquemático, de la argumentación que Derrida esgrime contra Husserl hay que destacar, por un lado, sus tesis acerca de la pasividad como condición de la actividad sintética de temporalización $y$, por otro, la idea -abandonada en su radicalidad posteriormente- de una génesis no trascendental y, por tanto, de una constitución dialéctica del objeto temporal. ${ }^{20}$ Este será, entonces, el antecedente para abordar la cuestión del

\footnotetext{
${ }^{19}$ Siguiendo a Paola Marrati-Guénoun, Derrida explora la filosofía genética de Husserl como la tesis de Levinas sobre una alteridad absoluta de modo de proponer una efectiva de entender, no solo el significado del tiempo, sino sobre todo el tiempo del significado (MARRATI-GUÉNOUN, 1998, p. 11).

${ }^{20}$ Lo que Derrida abre en este texto de 1956, publicado en 1990, es que habría una aporía en el corazón mismo de lo trascendental, toda vez que para pensar un origen absoluto del mundo es necesario postularlo como estrictamente no-mundano y no-existente. $\mathrm{Y}$ es que la fenomenología requiere que este origen no sea ni absolutamente no-mundano ni absolutamente mundano, sino que exige pensar
} 
lenguaje como escritura, primariamente a partir del problema del signo y su relación con la muerte, es decir, como estructura esencialmente finita que opera como testamento y testimonio de la muerte ineludible del sentido y de la subjetividad, muerte que es justamente lo otro en conflicto con lo mismo, con la vida como mismidad. Mediante la exposición de la adopción temprana en esta discusión con Husserl (El origen de la geometría, 1962; La voz y el fenómeno, 1967) del concepto de escritura, se explicitará la suplantación total que dicho concepto lleva a cabo respecto del lenguaje en general.

Lo específico de esta lógica de la contaminación que se descubre con Husserl, y lo que permite asociarla a una cierta violencia de origen, es que pone en entredicho el sistema legal de los textos filosóficos, la normatividad de sus principios y el privilegio jurídico asociado a éstos, desestabilizando así la soberanía asociada a la simplicidad del origen pensado desde lo que -desde Heidegger- puede señalarse como un gesto de reducción del tiempo al presente y a la presencia. Por esto, las constataciones sobre la violencia originaria se siguen en primera instancia de una reflexión y análisis del tratamiento del problema del tiempo en Derrida. Y esto en la medida en que aquello contra lo que se alza la violencia originaria es una cierta concepción del tiempo a partir del privilegio de uno de sus modos, el presente, y de una determinación del ente como ente-presente. Es necesario mostrar, entonces, cómo este privilegio de la presencia en la ontología, marcada por un reducción del devenir del tiempo mundano a estructuras trascendentales sincrónicas, ha forzado la institución de una legalidad discursiva y significativa que elimina, sin restos, toda su violencia intrínseca. En otras palabras, que niega taxativamente la posibilidad de pensar la anacronía del tiempo y la consecuente imposibilidad

una subjetividad no-humana, podríamos decir, absoluta, al tiempo que existente y congnoscible (LAWLOR, 2002, p. 18). De esta tensión propia de lo trascendental en sentido husserliano, nace en principio la necesidad en Derrida de pensar la cuestión de la génesis desde una cierta filosofía dialéctica, que no sea un simple método, sino que se plantee como «ontológica», es decir, que tenga su sustento en una cierta ontología temporal, irreductible a un formalismo puro. Esta base dialécticoontológica se opone sin duda a un análisis meramente estático e ideal de la cuestión del origen, examen que no sería justo siquiera con el mismo Husserl y su intento de escapar tanto del logicismo platonista como del empirismo psicologista. Pero dicha aproximación dialéctica no es plenamente adecuada a la idea de un origen histórico-trascendental, en la medida en que para la fenomenología debe pensarse en su simpleza y jerarquía: como no-divisible, no-complejo y como anterior -aunque sólo en sentido fundamental: de jure - respecto del origen mundano o histórico. La introducción de una dialéctica en el análisis (DERRIDA, 1990, p. 215 y ss.) no es así inocente: implica la intención de mostrar la imposibilidad misma de determinar el origen del movimiento dialéctico y, con ello, llegar al núcleo más problemático de la fenomenología para mostrar su inconsistencia. 
de una síntesis temporal perfecta como fundamento y origen de la constitución del sentido, de la verdad y la objetividad.

Un origen violento remite así a la mostración de una impureza inaugural, de una implicación irreductible con la alteridad a la que todo principio está sometido, que imposibilita la fijación de esa legalidad en su plena soberanía (DERRIDA, 1962, p. 14) y la revela en su debilidad constitutiva. En la medida en que esta contaminación, esta implicación con lo otro, ha sido pensada -desde aquel mismo privilegio legal-jurídico de la filosofía o metafísica de presencia- en su vínculo con el mal, la injusticia y, en suma, la violencia, impulsando así hacia una crítica generalizada de la misma, Derrida se vale de este concepto para señalar en sentido contrario: hacia una archiviolencia, violencia trascendental como condición del sentido -más allá del bien y del mal, por tanto- asociada a la dinámica de la differánce y la huella, es decir, hacia un origen genético del sentido que sólo puede plantearse, en su calidad de a priori que opera como -aunque sin poder realmente reducirse a ello- "condición" de posibilidad no clausurante, como cuasi-trascendental (pues sólo tiene efectos de trascendentalidad). Es la violencia como tensión que involucra a más de uno, como doble vínculo (double-bind) o como banda genética lo que está en juego en esta noción de un principio condicionante y estructurante, constituido en una implicación irreductible con lo empírico, cuya operación es la de una trascendentalidad que no puede ser simplemente trascendental. La misma formulación "violencia originaria" o "violencia trascendental" señalan en esa dirección, como a un origen que, en cuanto violento, no puede pensarse estrictamente desde la categorización del origen, desde su simplicidad.

Esta especificación de la violencia, como señalábamos, tiene su antecedente en la recepción de la fenomenología husserliana y el problema de la génesis, pero está aún más directamente relacionado con las discusiones que establece Derrida con otro fenomenólogo y "crítico" de la violencia: Levinas. La relación con el pensador lituano-francés no puede desestimarse en ningún punto respecto de la cuestión de la violencia. En su primer texto dedicado a él (Violencia y metafisica, de 1964 publicado en 1967) Derrida explora esos derroteros según los cuales la alteridad implica apertura no sólo a lo que aún no está aquí, en el presente, no sólo a lo que viene, sino también -y sobre todo- a quién viene. A un rostro, a un "usted". ${ }^{21}$ Apertura, dice Derrida, a cualquier/ ${ }^{21}$ Elemento clave en la filosofía de Levinas es la tercera persona, a la que le conviene el pronombre
usted. La relación yo-tú , dice Levinas, "excluye al tercero, permanece como intimidad, soledad de dos, 
radicalmente otro, la única forma de nombrar eso que en francés se resume tan sólo en la frase tout autre. La fenomenología genética será así la puerta de entrada para el pensamiento de lo otro que afecta a la conciencia y que impide, por eso mismo, una auto-afección de ella misma que sea totalmente identitaria. La no-identidad de esta auto-afección es también lo que permite su operación propia, la significación en cuanto tal, es decir, la constitución del sentido en cuanto siempre tensionado por lo sin-sentido, en cuanto referencialidad irresumible en un "yo pienso" absoluto. Es el lenguaje como lo constitutivo del sentido lo que finalmente debe entenderse como violencia. El polemos originario, la co-implicación, la tensión y el doble vínculo son, en último término, determinaciones del lenguaje en toda su extensión. Vale decir, en Derrida, del lenguaje como escritura. Levinas será así para Derrida, y no sólo desde esta temprana época textual, sino sobre todo en escritos muy posteriores (Palabra de acogida, 1996) la mediación necesaria para acoger a Husserl, y sentará así las bases de lo que ha de entenderse por violencia originaria, que en este primer apartado explicaremos a partir de dos ideas fundamentales, tomadas de ambos pensadores: sustitución e iterabilidad, resumidas en la cuestión, muy difícil de pensar, de la unicidad como lo inmediatamente iterable. $^{22}$

Si fuese posible sistematizar la relación en torno a la violencia de Derrida con Levinas, habría que al menos dar cuenta del alcance que el texto de 1964 Violencia y metafisica. Ensayo sobre el pensamiento de Emmanuel Levinas tuvo en la filosofía de Derrida, notando:

1. Que es el primer gran texto de Derrida sobre la violencia, pues aunque simultáneo a la publicación de De la grammatología, es de escritura

sociedad cerrada, lo no-público por excelencia” (1988, p. 297). El tercero presente en el rostro es lo que posibilita mi compromiso no sólo con un otro concreto -que puede ser mi amante o mi amigo, una relación privilegiada- sino con toda la humanidad. Por ello es que la sociedad entera es el lugar de la verdad, lugar dado por la obligación entregada por el otro como por un maestro. La relación es asimétrica, porque el otro es el absoluto y no está en el mismo plano que yo: no es tú, es usted.

${ }^{22}$ Cf. Derrida (2006b, p. 95-96), Cierta posibilidad imposible de decir el acontecimiento: "Es esa idea, muy difícil de pensar, de la unicidad como lo inmediatamente, como diría Levinas, comprometido en la sustitución. La sustitución no es simplemente el reemplazo de lo único irremplazable: la sustitución reemplaza lo irremplazable. Que haya inmediatamente, desde la primera mañana del decir o el primer surgimiento del acontecimiento, iterabilidad y retorno en la unicidad absoluta, en la singularidad absoluta, ello hace que la venida del arribante -o la venida del acontecimiento inaugural- no pueda ser acogida sino como retorno, (re)aparición, (re)aparición espectral." 
anterior $^{23}$. Sin embargo, es en este último donde el tema será articulado en directa relación al concepto de archi-escritura. En Violencia y metafísica Derrida por primera vez problematiza una cierta idea de violencia, y lo hace en plena continuidad con sus análisis anteriores sobre la fenomenología husserliana. Esto es visible en el tratamiento que hace del concepto a partir de las cuestiones sobre la fenomenalidad y el lenguaje, entre otras.

2. Pero también es posible establecer aquí un momento genético en el que emergen nociones que marcarán desde entonces el texto derridiano, y que configuran tesis fuertes y propias respecto de la violencia. Una de ellas, quizás la más importante, es la noción ya seńalada de economía de la violencia, ligada al aparecer del sentido y su circulación -capitalización infinita ${ }^{24}$; vinculada también, por lo tanto, con el tema más general del phainesthai, que es también el del fantasma, de la espectralidad del sentido en general, su vir-tualidad.

3. Por último, dar cuenta también que todos los desacuerdos con Levinas emergerán de esta nueva base conceptual con la cual operar el concepto de violencia: una cierta idea de lo jurídico en el método que no puede sin más erradicarse de los análisis de la fenomenología, y que será la que le permita conservar desde entonces el lazo indisoluble con ella, a través de

\footnotetext{
${ }^{23}$ Violencia y metafisica es publicado por primera vez en 1964, en Revue de Métaphysique et de Morale 69 (4):425 - 473. El ensayo citado de De la gramatología sobre Lévi-Strauss y Rousseau, está basado en un curso dictado por Derrida en la Ecole Normale Supérieure en el semestre de 1965-1966 bajo el título Ecriture et civilisation.

${ }^{24}$ La noción de economía se introduce en este texto para resistir la sustitución avasalladora de la historia tanto por la a-historicidad como por cualquier forma de ultra-historicidad -como la que Derrida parece detectar en Levinas (DERRIDA, 1967a, p.220). Dicha resistencia abre el campo no-totalizante de una economía de fuerzas, donde el cálculo y el programa económico-político se devela impotente para contener su naturaleza contenciosa. Esta noción de economía con la que Derrida trabaja está en relación con un pensamiento de la historicidad, que entiende la violencia de la relación con el otro como lo que permite salir de los cierres que la misma filosofía de la totalidad ha impuesto a la experiencia. A pesar de su lectura crítica, Derrida concede a Levinas el saber que la filosofía es "histórica de parte a parte" (historique de part en part) (DERRIDA, 1967a, p. 172), pues su crítica apunta tanto a la configuración de la experiencia como totalidad finita como a comprender la experiencia de la infinitud del otro como infinito positivo. En ambos casos, la historia se detiene, y la filosofía traiciona su propio compromiso tanto con la historicidad como con la finitud: con lo Otro, en suma. Pues si se trata de abandonar una filosofía de la totalidad, la justicia y la paz no pueden ser ya fines programables; deben desencadenar en el lenguaje una tendencia infinita, que lo obligue a reconocer y a practicar "una guerra en sí mismo. Violencia contra violencia. Economía de la violencia” (DERRIDA, 1967a, p. 172).
} 
una reflexión más general sobre la ley y el derecho, o más bien sobre la fuerza o la violencia propia de ambos. En suma, el concepto de violencia problematizado aquí por Derrida se vuelve a su vez productivo: de nuevos derroteros para la deconstrucción, que la arrancan de la conceptualidad estrictamente fenomenológica para ligarlos de manera más fuerte y directa con la esfera general de la ley, el derecho, la responsabilidad y la justicia.

La violencia en Levinas es siempre derivada, o segunda, posterior respecto de un momento original de no-violencia que es el encuentro con el otro, el cara-a-cara. En palabras de Bensussan (2008, p. 25): "Para que el gesto de prestar socorro tenga la consistencia de una práctica y la evidencia de su inteligibilidad, es necesario que sea dada una originalidad de rechazo a la violencia tan antigua como la violencia misma, y quizás más antigua." La violencia para Levinas es también siempre violencia de contra-golpe, se despliega cuando la impotencia se manifiesta, mostrando su absurdo, su paradoja: ella es siempre frustración, intento de totalización de lo in-totalizable, de dominación de lo que no se deja dominar ni encerrar en las categorías del ente -"el Otro es el único ser al que puedo querer matar." (LEVINAS, 1988a, p. 173). La violencia es así el rechazo, la reacción alérgica respecto del mandato del otro, rechazo que, sin embargo y paradójicamente, ya supone el encuentro con el otro, su recibimiento hospitalario por parte del Mismo y la alteración ética de la subjetividad o responsabilidad (BENSUSSAN, 2008, p. 29). Ya se ve aquí la inversión de la jerarquía entre la noción de ética y la de ontología que maneja Levinas: es el hecho de la relación con el otro hombre, en el sentido de su extrema singularidad y unicidad no-repetible, lo que adquiere aquí prioridad sobre el derecho de la comprensión, del aparecer en general o fenomenalización.

Lo anterior implicaría una constante soberanía de lo otro, que es matizada por una dualidad paradójica de la violencia: aunque el otro se presenta desde una dimensión de altura que lo hace intocable, como un maestro que habla en imperativo, el rostro también me revela esa fragilidad esencial que lo hace susceptible de violencia y asesinato. "La piel del rostro es la que se mantiene más desnuda, más desprotegida [...] El rostro está expuesto, amenazado, como invitándonos a un acto de violencia. Al mismo tiempo, el rostro es el que nos prohíbe matar." (LEVINAS, 2000, p. 72). El más allá del rostro está en constante contradicción con su encarnación y mundanización, pues siempre en su faz el otro es visto y recibido como el necesitado, como el más vulnerable 
de ser violentado, pero, al mismo tiempo, con un cierto cariz de soberanía que desde su asimetría y elevación me da la ley primera. La doble experiencia de la inspiración y obsesión ${ }^{25}$ que provoca su altura, por un lado, y la del horror, de la pobreza, del dolor en toda su concretud histórica, que se siguen de su vulnerabilidad, por otro, priman sobre la inclinación violenta, lo que hace de mí un ser respondiente del otro, pues no puedo callar ante el ruego (cri) del otro, ante la necesidad del otro y su petición de ayuda; el ruego se transforma así para mí en un mandato. Siendo el mandato principal de la ética el "no matarás", pues es la primera palabra del rostro (LEVINAS, 2000, p. 75), todo intento de reducción de su alteridad se opone a ese imperativo que me constituye como sujeto responsable de manera aún más originaria que las estructuras ontológicas de la existencia. La ética levinasiana supone una normatividad desplazada, que va de las estructuras intencionales de la conciencia al encuentro con el otro, de la esfera de los principio que solía ostentar el derecho al hecho mismo del encuentro infinitamente singular con otro.

Así, en la filosofía de Levinas la ontología sería constante presa de su propia traición a la alteridad, pues dicha traición trae consecuencias nefastas para el pensamiento, como el encierro eterno en la soledad del existir, el solipsismo y la imposibilidad de explicar el fenómeno del tiempo (DERRIDA, 1967a, p. 135-136). Derrida nota aquí el resabio de un sutil anti-intelectualismo -muy bien disimulado por el peso de las investigaciones fenomenológicas de Levinas ${ }^{26}$-, que plantea la total debilidad de una razón imposibilitada y destituida de su mismo ejercicio, y dependiente por ello del encuentro con el otro. La alteridad pasivamente recibida limita los poderes teóricos y prácticos de esta razón, que quedan desde el encuentro cara-acara desmentidos como principio. Entra en escena la idea de una crítica de la libertad, que hay que entender primariamente en términos especulativos: una crítica de la espontaneidad del pensamiento, de ese "yo pienso" que en Levinas, recordémoslo, se convierte en un "yo puedo", "en una apropiación de lo que es, en una explotación de la realidad” (LEVINAS, 1988, p. 16). Para que la libertad no sea violenta es necesario ponerla en crisis mediante una crítica que contiene la estructura original de la ética. La necesidad de una crítica de esta libertad, que la acuse y la llame a la responsabilidad, al respeto

\footnotetext{
${ }^{25}$ Estos conceptos aparecen posteriormente en la obra de Levinas, en De otro modo que ser o más allá de la esencia, de 1978 (LEVINAS, 2008, p. 173-188).

${ }^{26}$ En efecto, Levinas parece ser crítico del anti-intelectualismo, pues así lo manifiesta en relación con Heidegger, que al erradicar la intencionalidad de la fenomenoogía tropezaría con ese problema. (LEVINAS, 1988, p. 111).
} 
absoluto y generosidad frente a la alteridad es una de las tesis centrales de Levinas: justificar la libertad, no consiste en probarla, sino en hacerla justa (LEVINAS, 1988, p. 55). Y esta justificación solo puede ser dada a la libertad por algo que le es exterior y que irrumpe en medio de su ejercicio, en medio de la ontología. La justificación viene, entonces, sustentada por un derecho dado a lo exterior, a lo radicalmente otro.

Derrida continúa en Violencia y metafísica atento estos movimientos que, al igual que el espiral de Husserl que es citado por Derrida (1962, p. 14) en Introduccion a "El origen de la geometría" de Husserl, van de un lado a otro del límite -en zig-zag- que separa lo interior de lo exterior: lo interior del ser, de la conciencia y la existencia, de la historia también, hacia el exterior de la alteridad, hacia la exterioridad absoluta que supone la relación con el otro. De ahí que la complicación de lo jurídico aparezca nuevamente:

Retornar, como al único punto de partida posible, al fenómeno intencional en el que el otro aparece como otro y se presta al lenguaje, a todo lenguaje posible, es quizás entregarse a la violencia, hacerse cómplice al menos de ella y dar derecho - en el sentido crítico-a la violencia del hecho. Pero se trata entonces de una zona irreductible de la facticidad, de una violencia originaria, trascendental, anterior a toda elección ética, supuesta, incluso, por la no-violencia ética. (DERRIDA, 1967a, p. 183-184; terceras cursivas nuestras).

El lenguaje hace desde siempre que se pierda el sueño de la presencia de lo otro, presencia incapaz de aparecerse de otra manera que en su propia desaparición. En ese sentido, no solo la metafísica -o la ontología- es violenta. Todo aparecer de lo otro frente a mí, aunque sea en forma de presencia epifánica o escatología - palabras de Levinas-, supone ya esta violencia primera, que encierra a lo otro en la unidad de una experiencia. La fenomenología de Levinas niega una violencia que ya supone, la violencia de la fenomenalidad misma, del aparecer en general (DERRIDA, 1967a, p. 179-180) ${ }^{27}$, ya trastocado y dislocado - dislocante también- de lo otro, de la experiencia del cara-a-cara. El otro aparece ya en ese encuentro en cuanto sujeto de un discurso que, de hecho $y$ de derecho, está ya implicado en la fenomenalidad (TIMM DE SOUZA,

${ }^{27}$ Dice Derrida que hay dos sentidos de lo teorético, en cuanto objeto de cuestionamiento del discurso de Levinas: el primero es el sentido "corriente", como teoría que se opone y jerarquiza frente a la sensibilidad, que se impone y violenta toda receptividad de la conciencia y, por ende, que neutraliza toda pasividad. El segundo, sentido "oculto", es el del aparecer en general, en el cual también tiene lugar lo no-teorético - derivado del primer sentido- en particular. "En este segundo sentido la fenomenología es por cierto un teoreticismo, pero lo es en la medida en que todo pensamiento y todo lenguaje, de hecho y de derecho, están ligados al teoreticismo." 
2009) ${ }^{28}$-aunque sea una fenomenalidad, sin duda, "fantasmática”. Derrida se refiere a esta violencia, en este lugar específico, como violencia trascendental, justamente en cuanto la violencia está supuesta a priori como condición de posibilidad del sentido, como la lógica de su normatividad. Esta idea y este nombre dado a la violencia - "trascendental" - no se repetirá en ningún otro texto de Derrida, y sin embargo señala un matiz que será desde ya insoslayable por las reflexiones sobre la violencia.

SALVATERRA, V. C. Archi-violence: the genesis of the violence of genesis in Derrida's philosophy. Trans/form/ação, Marília, v. 42, n. 4, p. 99-124, Out./Dez., 2019.

\begin{abstract}
In this paper, I approach the concept of violence in connection with deconstruction as a condition of possibility of meaning. I track the genesis and structure of different formulations of the concept presented in the early works of Derrida (1954-1967), such as 'original violence', 'transcendental violence', and 'archi-violence'. I present some key points on this topic emphasizing two features: 1) the overall thesis about the economy in which violence is exposed and developed, in dialogue with the ideas of Lévi-Strauss; and 2) some more detailed notes about the genesis of the concept of violence, which is adopted by Derrida in direct discussion with the phenomenological tradition, especially with Husserl and Levinas. Both subjects are also involved with certain methodological elements that I think
\end{abstract}

\footnotetext{
${ }^{28}$ Sobre la ética como filosofía primera: "O está la dignidad del Otro en el origen de todo el pensamiento, pensamiento que deriva de la relación humana sin la cual nada de lo que es humano puede ser comprendido, $o$ el Otro concreto es rápidamente metamorfoseado en otro meramente lógico, reproduciendo la relación de violencia más posible de encontrar no solo en la historia del mundo, sino también en la historia del pensamiento: estas primas tan próximas." (TIMM DE SOUZA, 2009, p. 237). Respecto de estas afirmaciones, habría que preguntarse: ¿qué es la dignidad del otro? ¿no supone cualquier concepto de dignidad o de respeto un reconocimiento? Y sobre "meramente lógico", sin duda refiere a un sentido tradicional, al sentido "propio" de lo lógico, que no sería sostenible desde la tesis de la polémica originaria de lo otro en lo mismo. Proponemos pensar lo otro como otro en general, pues ese "en general" tendría ya la marca de una diferencia irresumible, sería un concepto abierto, autodiferido, imposible de clausurarse en la lógica clásica. Por ello, tampoco sería lo ilógico o lo a-lógico por excelencia, toda vez que la estructura de lo otro en lo mismo o de la sustitución implican ya una cierta lógica, aunque desbordada por lo otro dentro de sus propios límites.

Esto se refuerza cuando Timm de Souza dice luego que el núcleo de la violencia está en "la sustitución del otro por su mero concepto -un concepto mudo, sin lenguaje, cómplice de la injusticia y que abre finalmente espacio para las sustituciones de la temporalidad real por sus figuraciones lógicas, en el completarse tautológico de la Totalidad" (TIMM DE SOUZA, 2009, p. 240). Nuevamente, se habla de "el mero" concepto, sin tener en cuenta la posibilidad, instaurada por el mismo Levinas, de un concepto diferido, autodiferido del otro, según el cual el otro puede afectarme como otro, ser reconocido por mí e investirme como responsabilidad.
} 
cannot be avoided in Derrida's philosophy, because they are integral to deconstruction itself. This will give us a full view of the problem of the idea of a genesis of the violence of genesis.

KEYwords: Violence. Derrida. Genesis. Economy. Phenomenology.

\section{REFERENCIAS}

BEARDSWORTH, R. Derrida y lo politico. Buenos Aires: Prometeo, 2008.

BENJAMIN, W. Para una crítica de la violencia. In: OYARZÚN P.; PÉREZ C.; RODRÍGUEZ, F. Letal e incruenta: Walter Benjamin y la crítica de la violencia. Traducción y notas de Pablo Oyarzún. Santiago: LOM, 2017.

BENSUSSAN, G. Ethique et expérience: Levinas politique. Strasbourg: La Phocide, 2008.

CAMPOS, V. Husserl qui genuit Levinas, qui genuit Derrida: lo jurídico y la violencia genética. Revista Actuel Marx, n. 14, p. 281-208, 2013.

CAMPOS, V. Violencia y fenomenología. Derrida, entre Husserl y Levinas. Santiago: Metales Pesados 2017.

CRITCHLEY, S. The ethics of deconstruction: Derrida and Levinas. Edinburgh: Edinburgh University Press, 1999a.

CRITCHLEY, S. Ethics-politics-subjetivity: essays on Derrida, Levinas and contemporary French thought. London-New York: Verso, 1999b.

CRITCHLEY, S.; BERNASCONI, R. (ed.). Re-reading Levinas. Bloomington: Indiana University Press, 1991.

CRITCHLEY, S. ; DEWS, P. (ed.) Deconstructive subjectivities. Ithaca, NY: State University of New York Press, 1996.

DERRIDA, J. Introducción a "L’origine de la géométrie" de Husserl. Paris: Presses Universitaires de France, 1962.

DERRIDA, J. L'ecriture et la difference. Paris: Seuil, 1967a.

DERRIDA, J. De la gramatologie. Paris: Minuit, 1967b.

DERRIDA, J. Marges de la philosophie. Paris: Minuit, 1972.

DERRIDA, J. Psyché. Inventions de l'autre. Paris: Galilée, 1987.

DERRIDA, J. Le problème de la genèse dans la philosophie de Husserl. Paris: Presses Universitaires de France, 1990.

DERRIDA, J. Donner le temps. 1: la fausse monaie. Paris: Galiée, 1991.

DERRIDA, J. Espectres de Marx: l'état de la dette, le travail. Paris: Galilée, 1993a.

DERRIDA, J. La voix et le phénomène. Paris: PUF-Quadrige, 1993b. 
DERRIDA, J. Politiques de l'amitie. Paris: Galilée, 1994.

DERRIDA, J. Adieu à Emmanuel Levinas. Paris: Galilée, 1997a.

DERRIDA, J. Le mot d'accueil. In: Adieu à Emmanuel Levinas. Paris: Galilée, 1997b.

DERRIDA, J. El monolingüismo del otro. Traducción de Horacio Pons. Buenos Aires: Manantial, 1997c.

DERRIDA, J. Canallas: dos ensayos sobre la razón. Traducción de Cristina de Peretti. Madrid: Trotta, 2005.

DERRIDA, J. Fuerza de ley: el fundamento místico de la autoridad. Traducción de Adolfo Barberá y Patricio Peñalver. Madrid: Tecnos, 2008a.

DERRIDA, J. Marx \& Sons. In: SPRINKER, M. (ed.). Ghostly Demarcations: a symposium on Jacques Derrida's Specters of Marx. London: Verso, 2008b. p. 213-269. GARRIDO, J. M. Chances de la pensé: à partir de Jean-Luc Nancy. Paris: Galilée, 2011. GRANEL, G. Jacques Derrida et la reture de l'origine. In: Traditionis traditio. Paris: Gallimard, 1972. p. 154-175.

LAWLOR, L. Derrida and Husserl: the basic problem o phenomenology. Bloomington: Indiana University Press, 2002.

LEVINAS, E. Totalité et infini: essai sur l'extériorité. La Haye; Boston; Londres: Martinus Nijhoff, 1988.

LEVINAS, E. Ética e infinito. Traducción de Jesús María Ayuso Díez. Madrid: La Barca de la Medusa, 2000.

LEVINAS, E. Descubriendo la existencia con Husserl y Heidegger. Traducción de Manuel E. Vásquez. Madrid: Síntesis, 2005.

LEVINAS, E. Autrement qu'être ou au-delà de l'essence. Paris: Le Livre de Poche, 2008.

MALABOU, C. Économie de la violence, violence de l'économie (Derrida et Marx). Revue Philosophique, T. 180, n. 2, p. 303-324, 1990.

MARRATI-GUÉNOUN, P. La Genèse et la trace. Derrida lecteur de Husserl et Heidegger. Dordrecht: Kluwer Academie, 1998.

MARSH, J. Of violence: the force and significance of violence in the early Derrida. Philosophy Social Criticism, v. 35, n. 3, p. 269-286, 2009.

NIELSBERG, J.-A. Jacques Derrida, penseur de l'évènement. Entretien avec Jacques Derrida. L'Humanité, 28 janvier, 2004. Disponibele en: http://www.humanite.fr/ node/299140. Accès en: 20 mai 2018.

PEETERS, B. Derrida. Traducción de Gabriela Villalba. Buenos Aires: Fondo de Cultura Económica, 2013

RAMOND, C. Matérialism et hantologie. Cités, v. 2, n. 30, p. 53-63, 2007. 
SALVATERRA, V. C.

TIMM DE SOUZA, R. El pensamiento de Levinas y la filosofía política: un estudio histórico-filosófico. In: BONZI, P.; FUENTES, J.J. El énfasis del infinito: esbozos y perspectivas en torno al pensamiento de Emmanuel Lévinas. Santiago de Chile: Antrhopos; Universidad de Chile, 2009 .p. 225-242.

Recebido: 13/06/2017

Aceito: $29 / 11 / 2018$ 